\title{
El problema de la delimitación o boundary problem. Una aproximación a la definición de la clase media
}

\author{
Modesto Gayo \\ Escuela de Sociología, Universidad Diego Portales, Santiago, Chile. \\ Email: modesto.gayo@udp.cl
}

\begin{abstract}
Resumen: El declive del interés en la perspectiva de clase en ciencias sociales como enfoque para la explicación de los fenómenos sociales no ha ido acompañado de la total desaparición del vocabulario de clase. En efecto, en la actualidad es cada vez más habitual encontrarse frente a debates sobre la clase media tanto dentro de la academia como en el área del comentario social. En este sentido, la clase media es considerada expresión no sólo de una realidad social, sino de un deber ser político. Aunque el término clase media es utilizado con mucha frecuencia, más allá de los textos sociológicos, este artículo enfatiza que no ha existido acuerdo entre académicos sobre su definición, lo cual ha impedido trazar de forma consensuada y unánime fronteras entre esta clase y las otras. Este ha sido conocido como el boundary problem o, como lo traducimos aquí, el problema de la delimitación de las fronteras de clase. El objetivo de este artículo es presentar este problema en toda su complejidad, y por ello hablamos de su definición, las condiciones intelectuales en que emerge, sus causas, y las soluciones que se han adelantado.
\end{abstract}

Palabras clave: clase media, estratificación, clase social, problema de la delimitación de fronteras de clase social, análisis de clase.

\section{The boundary problem. Towards a definition of middle class}

\begin{abstract}
The declining interest in class analysis in social sciences as an approach which contributes to the understanding of social phenomena has not implied the complete extinction of class lexicon. Indeed, it is increasingly common to find debates about the middle class, not only within the academia but also at the social commentary level. Thus, the middle class is considered an expression not only of a social reality, but of a political ideal too. Even though the term middle class is frequently used, beyond sociological research, this article stresses that there has not been agreement between academics about its characteristics, and this has made difficult to draw widely accepted borders between this and other classes. This has become known as the boundary problem. The aim of this paper is to offer a comprehensive view of this problem, particularly focusing on debates about its definition, intellectual conditions of emergence, causes, and some suggested solutions.
\end{abstract}

Key words: middle class, stratification, social class, boundary problem, class analysis. 


\section{O problema da demarcação ou problema de fronteira. Uma abordagem para a definição da classe média}

Resumo: O declínio do interesse na perspectiva de classe da ciência social como uma abordagem para a explicação dos fenômenos sociais não tem sido acompanhado pelo desaparecimento total do vocabulário de classe. Na verdade, hoje em dia é cada vez mais comum encontrar com as discussões da classe média, tanto dentro da academia e na área de comentário social. Neste sentido, a classe média não é apenas considerada uma expressão de uma realidade social, mas de um dever ser político. Embora o termo «classe média» é usado muitas vezes para além dos textos sociológicos, este artigo enfatiza que não houve acordo entre os estudiosos sobre a definição, que tem mantido enredo por ausência de consenso e unanimidade dos limites entre esta classe eas outras. Isto tem sido conhecido como o " problema de fronteira «, ou, como aqui traduzido, o problema da demarcação dos limites da classe. O objetivo deste artigo é apresentar o problema em toda a sua complexidade e, portanto, falamos de sua definição, as condições intelectuais em que surgem, suas causas eas soluções que foram apresentadas.

Palavras-chave: classe média , estratificação , classe social, com problemas de delimitação das fronteiras de classe social, análise de classe .

$* * *$

\section{Introducción al problema de la delimitación desde el análisis de clase}

Parece que en la sociología de principios del siglo XXI, el estudio de los esquemas de clase perdió interés. En el mejor de los casos, cuando el interés pervive, para aquellos investigadores con formación en la literatura anglosajona bastaría elegir dentro del menú ofrecido, el cual tiene dos platos principales, J. Goldthorpe (Erikson y Goldthorpe, 1993) y E.O. Wright $(1983,1994)$. En el peor de los casos, en lo que al uso de la clase se refiere, el interés se evaporó en el mismo momento en que el marxismo cayó en desuso político y en descrédito intelectual ${ }^{1}$. Si las clases no vivían enfrentadas y la política no era un epifenómeno de la lucha o el conflicto de clase para qué continuar escribiendo y pensando sobre algo que no existe en un mundo crecientemente individualizado (Bauman, 2001 y 2007; Beck y Beck, 2002). Puede parecer paradójico pero el declive del análisis de clase dejó un legado incólume y más fuerte que nunca, dado que se considera expresión del hombre y la mujer promedio que constituyen el cuerpo social, la clase media ${ }^{2}$. En otros términos, la sociedad en la que desaparecen las contradicciones de clase, apuntará hacia un futuro promisorio del que la clase media será su representante (Gayo et al., 2012) .

Sin embargo, a pesar de haber ganado terreno en muchos países la construcción de una ampliamente compartida subjetividad de clase media ${ }^{4}$, ha seguido siendo patente que las desigualdades continúan, lo que finalmente ha exigido nuevamente recurrir a esquemas, principalmente los ya disponibles, que representan tal realidad social (Franco et al., 2007). 
Este artículo reflexiona teóricamente sobre las dificultades que han existido para identificar o definir a la clase media y, en relación con ello, problematiza el uso de esquemas de clase construidos sobre la base de casos particulares y en la actualidad motivo de investigaciones realizadas en el pasado, cada vez más lejano. En definitiva, si queremos hacer uso de las clasificaciones de clases sociales no deberíamos hacerlo de forma acrítica, y si estamos dispuestos a hablar de la clase media, conviene conocer la problemática que está implicada en su uso ${ }^{5}$.

Esta dificultad en la definición de la clase media se hizo conocida como el problema de la delimitación. Sin embargo, aunque los estudios se referían principalmente a esta clase, las reflexiones que llevaron al desarrollo de esta problemática, al igual que sus implicaciones, no pueden circunscribirse únicamente a aquélla. Como problema, su afectación es general, y muchas de las observaciones que presentaremos podrían aplicarse a otras clases. En definitiva, el boundary problem está íntimamente relacionado con la construcción de los esquemas de clase.

Adicionalmente, aunque lo más común ha sido la fabricación y uso de esquemas de clase referidos al ámbito del trabajo o las ocupaciones, lo que implica trabajar con clases ocupacionales, los problemas relativos a la definición o trazado de fronteras entre las clases no se derivan del hecho mismo de tratar con ocupaciones sino de la propia constitución de los esquemas. Esto quiere decir, por un lado, que definir clases basadas en otros criterios (por ejemplo: los estilos de vida, el consumo cultural, los ingresos $)^{6}$ nos enfrenta con problemas similares ${ }^{7}$. Por otro lado, significa que debemos atender con especial atención a la categorización misma. Por tanto, cualquier eventual solución satisfactoria debe valorar con particular detenimiento las categorías creadas y las posibilidades y límites de categorizar.

\section{A propósito de las dificultades de la definición de fronteras de la clase media}

Diferentes criterios han sido empleados para definir a la clase media, con el resultado de que no es siempre sencillo saber dónde están las fronteras sociales que este concepto promueve y, por tanto, de quién estamos hablando. Generalmente, algunos grupos ocupacionales han sido considerados parte de la misma (directivos, técnicos, profesionales), e igualmente no ha sido infrecuente excluir a algunos otros (empleados no manuales de rutina $)^{8}$. La dificultad que los diferentes autores han encontrado a la hora de definir a esta clase social, y los desacuerdos entre los mismos que ello ha conllevado, se ha hecho conocida como el problema de la delimitación (the boundary problem). En realidad, podría hablarse de un doble problema, pues ha implicado dos cuestiones ligadas entre sí, las que tratamos a continuación. 


\section{Una dificultad para encontrar qué tienen en común los individuos y grupos que componen esta clase social}

Evidentemente, el hecho de que no sea fácil encontrar aspectos compartidos está directamente conectado con la discusión sobre su heterogeneidad interna (Crompton, 1992) y el que podría ser llamado el boundary problem en su interior. No es raro, por tanto, que se hable en plural, comúnmente haciendo uso del término clases medias. Un buen ejemplo de la defensa de esta diversidad dentro de la unidad lo encontramos en Brint (1985), el cual afirma que en el estrato de profesionales podemos hallar importantes fracturas políticas de acuerdo con la ocupación, la cohorte y el tipo de organización en la que los individuos están empleados.

En un sentido diferente, siguiendo a F. Devine (1997), el hecho de que se hayan sugerido diferentes formas de diferenciación dentro de la clase media plantearía dudas sobre la posibilidad de identificar una entidad económica, social y política distintiva. Y concluye que no estamos ante una entidad socio-política coherente ${ }^{9}$. Otros autores en la misma línea serían Pilbeam (1990) y Cohen y Howard (en Walker, 1979). Si a la idea de pluralidad le añadimos una perspectiva histórico-temporal o dinámica, podemos sugerir que estamos ante un proceso de creciente (Abercrombie y Urry, 1983; Savage, Dickens y Fielding, 1988; Hanlon, 1998; Bosc, 2008) o decreciente (Goldthorpe, en Butler y Savage, 1995) fragmentación de este grupo social.

En cualquier caso, cualesquiera que sea el diagnóstico y el pronóstico, el debate sobre su heterogeneidad y las implicaciones de la misma está servido. Se observa, por tanto, que es difícil hallar un criterio universal que identifique a los grupos que pertenecen a la nueva clase media (Lederer y Marschak, en Vidich, 1995), y sería precisamente este hecho el que dotaría al debate sobre las fronteras de las clases medias de una relevancia especial (Howe, 1992) ${ }^{10}$.

\section{Una dificultad para delimitar con claridad lo que tienen de diferente con respecto a las otras clases}

En sentido estricto, este ha sido el que ha venido a ser conocido como problema de la delimitación de las fronteras de clase. Estamos ante un hecho central que se ha visto expresado tanto en las diferentes conceptualizaciones de las clases medias como en las soluciones que se han dado al problema de la delimitación, haya sido éste hecho explícito o no. A modo de ejemplo, Barbara y John Ehrenreich (en Walker, 1979) afirman que las fronteras que separan a la clase de profesionales y directivos (CPD) de las clases dirigente y trabajadora son borrosas. Raynor (1970) se manifiesta en el mismo sentido, aunque refiriéndose a la clase media. Esta afirmación es especialmente significativa porque los intentos de definir con claridad a la clase media han pasado habitualmente por negar su pertenencia a la clase trabajadora, o, en otros términos, por destacar sus diferencias con respecto a la misma (Bonham, 1954; Garrard y Parry, en capítulos dife- 
rentes, en Garrard et al., 1978; Savage et al., 1995).

Parry (ibídem) añade a este hecho la idea de que la clase media también sería la que quedaría después de que la clase alta o dirigente fuese definida o identificada. En este sentido, podemos mencionar dos respuestas bien distintas. Por un lado, Dahrendorf (1969) defiende que la nueva clase media ha resistido todos los intentos de definir sus límites superior e inferior $^{11}$; y en la misma línea estarían los Ehrenreichs (en Walker, 1979). Por otro lado, Goldthorpe (en Carabaña et al., 1994) afirma que por arriba encontraríamos individuos en posiciones de poder pero sin dejar de pertenecer a una clase de empleados y, por abajo, la divisoria la definiría la naturaleza del intercambio en la relación de empleo: la falta o existencia de recompensas prospectivas en términos de seguridad del estatus y la promoción.

Si a la metáfora espacial, implicada en palabras como arriba y abajo, agregamos un enfoque temporal, cabe incorporar una reflexión que piensa a las clases medias en perspectiva histórica. Esto es lo que sucede cuando Eder (1995) apunta que el concepto de nueva clase media plantea el problema de si puede ser visto como una continuidad de las viejas clases medias, lo que sitúa la discusión más allá de la diferenciación con respecto a las clases alta y trabajadora.

En conexión con esta idea, si bien la propiedad ha sido habitualmente considerada el criterio de separación de la clase media y las burguesías, grande y pequeña, en algunas ocasiones se ha sugerido que la primera podría formar un mismo grupo en conjunto con los pequeños propietarios (Poulantzas, 1974b; Brooks y Manza, 1997). Por abajo, con respecto a la clase trabajadora, dividen la autoridad, el conocimiento, las recompensas materiales y el estatus, siendo este último un criterio más genérico, a menudo inclusivo de los anteriores. En todo caso, en general, los criterios de delimitación se refieren a las ocupaciones, o a características particulares de las mismas, punto de partida habitual de la definición de las clases y elemento central en la construcción de explicaciones clasistas de los comportamientos tanto individuales como colectivos.

Reconociendo que ambos tipos de diferencias dentro de y entre las clases existen y, por tanto, que hay importantes dificultades para la delimitación de sus fronteras sociales objetivas, Devine (1997) sostiene que lo importante es determinar cuál de los patrones de diferenciación, intra- o entre-clases, es más relevante.

\section{Condiciones teóricas sobre las cuales el problema emerge}

Una vez mostrado que efectivamente la definición de la clase media ha sido una temática relevante en la sociología, y después de haber reconstruido la complejidad de dicha problemática, en esta sección analizamos los supuestos e intereses de los investigadores sobre los cuales se ha confi- 
gurado la delimitación de las fronteras de clase como un problema. En otras palabras, nos centramos aquí en elementos propios de los enfoques teóricos que ayudan a comprender la constitución intelectual de dicho asunto. Para ello, hacemos una distinción entre dos aproximaciones teóricas.

El primer enfoque estaría representado por aquellos autores que defienden el empleo de un esquema de clases para el estudio de las sociedades industriales avanzadas, las que en ocasiones también han sido conocidas como post-industriales, y cuya construcción tiene que ver con las características de las posiciones y/o relaciones en el trabajo. Estas contribuciones tienen una aproximación teórica al estudio del comportamiento de los individuos (Erikson y Goldthorpe, 1993; Wright, 1983 y 1994). Ello significa que se espera que los esquemas clasistas contribuyan a la explicación de los comportamientos individuales. Por tanto, se atiende principalmente a los criterios de configuración de las clases y la actuación de los grupos así definidos como clases sociales. El boundary problem, desde esta perspectiva, tiene que ver con la discusión en torno a aquellos criterios, y las principales preocupaciones son, por un lado, ubicar correctamente a los individuos en el esquema de clases y, por otro lado, prestar atención a la correspondencia entre el modelo de sociedad de clases y la realidad de la misma en términos de los comportamientos efectivos de los individuos clasificados, es decir, lo que significa atender a la eficacia explicativa de la clasificación de clases utilizada.

El segundo enfoque teórico al que nos queremos referir se centra en la formación de clases (Crompton, 1992; Wacquant, en McNall, 1991). Es parte central del mismo una aproximación empírica, o que depende de los hallazgos empíricos en cada circunstancia y momento histórico, y que por esa misma razón rechaza imponer categorías rígidas a la realidad. El problema de la delimitación de las fronteras de clase se produce tanto entre las clases como dentro de cada una de ellas, y es observado como una condición de la sociedad en la que vivimos, por tanto no superable, lo que precariza el uso de los esquemas de clase desde el principio y por definición. En este caso, se atiende a la multidimensionalidad que encierran los individuos, esto es, a su identidad, cultura, trayectoria, comportamientos, y otros que puedan ser de interés, todo ello imposible de reducir a un esquema de clases, y más si el mismo se centra exclusivamente en la esfera laboral. Los individuos son situados en primer plano y las clases sociales son un producto del actuar cotidiano y plural de los mismos, por lo que su comportamiento deja de ser pensado como una consecuencia directa e inmediata de su posición en la estructura social.

El boundary problem, en definitiva, va más allá de ser un mero producto de la dificultad conceptual para idear nítidas fronteras de clase, para devenir parte consustancial del enfoque teórico adoptado por el investigador. Sin duda, es visto como un problema por parte de los autores cuyo objetivo es contar con una clasificación de clases cuyos tipos sean lo más diferentes entre sí que sea posible, finalmente como si todo lo fundamental para los individuos ocurriese en una dimensión, la laboral, y en un momen- 
to en el tiempo, el del estudio o la encuesta realizados. En sentido contrario, la dificultad para delimitar clara y definitivamente las fronteras de clase es una condición de la realidad social, y no tanto un problema, para aquellos investigadores que entienden que las posiciones que adoptan los individuos tienen que ver con diferentes facetas de los mismos, y que sostienen que las clases, antes de que socialmente dadas, son producto de una constitución histórica contingente, frágil o fluida, cuyo orden tiene frecuentemente bases políticas y cuyo destino está siempre bajo amenaza.

\section{Causas de las dificultades en el delineamiento de la clase media}

En las secciones previas, hemos constatado la existencia de dificultades para definir con claridad las fronteras de la clase media con respecto a las otras clases sociales, e igualmente para dar respuesta a la pregunta de qué es lo que los miembros de este grupo tienen en común. Asimismo, hemos expuesto la relevancia del enfoque teórico para entender la constitución del boundary problem como un problema relevante para el pensamiento social. Sin embargo, todavía no tenemos las razones específicas que justifican dicha problemática. Esto es muy importante puesto que si pensamos en prestar atención a eventuales soluciones, es clave tener claridad sobre las razones o causas que conducen al problema de la delimitación. Tal y como aparecen en la literatura especializada, las causas son un grupo variado. Con el propósito de hacernos cargo de esa complejidad, a continuación ofrecemos un listado de las mismas que pretende ser tan exhaustivo como sea posible.

1. La inexistencia de un consenso sobre los criterios que definen a la clase media. Inglehart (1991) subraya este punto, si bien lo hace en referencia a la nueva clase. Dada la diversidad y cantidad de los criterios de definición (explotación, dominación y autoridad, relaciones de empleo, autonomía, capital cultural, educación, recursos, situaciones de mercado, propiedad, tipo de trabajo y función), no puede sorprender ni la afirmación sobre la falta de consenso, lo que se hace evidente en la pluralidad de aquéllos, ni las diferencias en el concepto de clase media. Ello exige a los investigadores que utilicen el concepto de clase media que hagan evidente en cada caso y circunstancia qué es lo que entienden por la misma, y asimismo les obliga permanentemente a atender a lo que otros quieren decir con su uso.

2. La complejidad de la estructura de clases contemporánea. Como producto de la misma, Burris (1987) arguye que nos encontramos con la ambigüedad de las posiciones de clase intermedias. Al hablar de complejidad, deberíamos entender que las posiciones individuales en la estructura social se definen de acuerdo a múltiples criterios o dimensiones, lo que dificulta describir grupos sociales muy diferentes de los otros. 
3. El cambio del significado a lo largo del tiempo (Bosc, 2008) ${ }^{12}$. De ello se derivan los obstáculos para alcanzar un uso continuado e históricamente compartido de la noción de clase media. Según Gunn (2004), ni el significado de cultura ni el de clase media fueron estables y continuos, y solamente a principios del siglo XX adoptaría el segundo término una definición sociológica moderna. Por tanto, podríamos decir que el momento histórico y el enfoque parecen haber sido relevantes a la hora de abordar la definición de la clase media. Adicionalmente, a ello podríamos agregar, con Devine (2011), la significación de las diferencias en el espacio o a nivel comparado.

4. El significado como producto de una constante lucha entre actores. Desde este punto de vista, el contenido del término clase media dependerá del resultado de los enfrentamientos entre grupos por ocupar las posiciones que caracterizan a la clase social aludida. El hecho de que un grupo de actores sean o no parte del mismo podría ser entendido como una contingencia histórica, consecuencia del éxito o fracaso alcanzado en su lucha por dar satisfacción a sus intereses de mantener o mejorar su situación en la sociedad de la que forman parte. Hanlon (1998) contribuye a personificar esta explicación al observar que el profesionalismo es actualmente un área de lucha entre importantes actores económicos ${ }^{13}$. Este proceso conflictual podría afectar tanto a los individuos y ocupaciones implicadas, o a las características significativas de los mismos, como a los criterios que definen la pertenencia a las diferentes clases sociales.

5. El continuo cambio en composición y función. Como resultado, siguiendo a Raynor (1970), nos encontramos con que las fronteras de la clase media con las clases alta y trabajadora son borrosas. Estamos ante una perspectiva dinámica que deja espacio para las tesis incluidas en los puntos 3 y 4, el cambiante significado del concepto de clase media a lo largo de la historia y la comprensión del mismo como un producto de enfrentamientos continuados entre grupos sociales, respectivamente.

6. La movilidad entre clases sociales. El hecho de que existan posiciones entre las clases o en los márgenes de las mismas en las cuales se observa una importante movilidad social (Abercrombie y Urry, 1983) dificulta la definición de sus fronteras. Es la movilidad individual entre posiciones incluidas en diferentes clases lo que complica adoptar una decisión sobre la pertenencia de clase de las personas que han experimentado ese desplazamiento. En otros términos, la movilidad social hace menos nítidas las consecuencias de pertenecer a una clase social concreta. Desde otro punto de vista, se esperaría que un mismo esquema o clasificación de clases aplicado a los individuos que no sufren movilidad social debería incorporar fronteras de clase visibles y con efectos claros. Ello quiere decir que las historias individuales tienen efectos en la capacidad de los esquemas de clase para explicar fenómenos cualesquiera, pero es necesario dejar claro que los criterios de definición de las clases no se ven necesariamente afectados por la movilidad que los individuos puedan experimentar. 
7. Las posiciones intermedias como posiciones de paso. Bourdieu (1979) es el que caracteriza a las posiciones medias o centrales como lugares de paso. De esta manera, se les resta entidad como posiciones y son presentadas de una forma residual, como un camino hacia una situación que sí define la pertenencia a una clase social en sentido estricto, y por tanto con todas sus consecuencias.

\section{Soluciones al problema de la delimitación}

Si la delimitación de fronteras de clase ha sido identificado como un problema, no debe sorprender que se hayan ofrecido soluciones al mismo. En realidad, podemos argumentar que nos encontramos ante un doble problema, pues se trata de definir los límites de una clase social, la clase media, que algunos autores consideran problemática (Wright, 1994). A este respecto, Sørensen (en Crompton et al., 2000) entiende a las clases medias como un problema e indica que cualquier eventual propuesta de un concepto de clase debe mostrar que puede dar respuesta al mismo. En todo caso, sin insistir en problematizar a esta clase social, es claro que estamos ante un grupo que está aquí para quedarse, que no es un fenómeno transitorio, lo que reclama un entendimiento cabal de la misma. Con el objetivo de avanzar en esta dirección, en este apartado concentramos nuestros esfuerzos en exponer algunas de las soluciones más importantes que se han ofrecido al problema de la delimitación, haya sido éste reconocido o no.

Dos son los tipos de propuestas que han tenido como objetivo proporcionar una solución al problema de la definición de fronteras de clase, a las que aquí denominaremos teórica y empírica. Esto significa, como exponíamos en la sección 3 o sobre las condiciones, que el enfoque teórico incide directamente en el tipo de solución que se propone.

\section{Solución teórica}

Esta salida incorpora aportaciones diferentes, como las clásicas de Goldthorpe (Erikson y Goldthorpe, 1993) y Wright $(1983,1994)$. No obstante, destaca la presencia de autores que pueden ser ubicados dentro de la tradición de pensamiento marxista (Poulantzas, 1974; Wright; 1983, 1994; Callinicos y Harman, 1987), lo cual tendrá una repercusión directa en sus afirmaciones, pues estarán más pendientes de encajar sus ideas dentro del acervo teórico derivado de tal escuela que de prestar atención en primera instancia al comportamiento de los individuos pertenecientes a la clase media, cuya existencia en dicha tradición de pensamiento es habitual motivo de debate. Desde un punto de vista teórico, podemos destacar cuatro soluciones, estando las tres primeras ligadas al marxismo.

1. Una primera forma de resolver el problema es negar la existencia de la clase media. La tesis de la proletarización de sus condiciones de trabajo puede ilustrar esta idea (Braverman, 1974), si bien este mismo autor parece aceptar que en algún momento hubo un grupo distinto tanto de la 
burguesía como de la clase trabajadora. En definitiva, esta vía significa que ya no hay fronteras que trazar entre la clase media y la obrera, pues en las nuevas condiciones del mundo del trabajo la primera deviene parte de la segunda, es decir, la clase media se convierte en proletariado.

2. La segunda solución pasa por privar a los miembros de la clase media, o a algunos de ellos, de una posición de clase (Wright Mills, 1959; Poulantzas, 1974b; Raynor, 1970; Cohen y Howard, en Walker, 1979; Callinicos y Harman, 1987). Por tanto, sin negar la existencia de un grupo peculiar que no puede ser incorporado en ninguna de aquellas dos clases (burguesía y proletariado), no se le reconoce un estatus de clase social, lo que en caso contrario para estos autores implicaría redefinir el mapa social de intereses y actores, $y$, en consecuencia, la forma de pensar en torno a la conciencia de clase, la organización política de las clases y, en definitiva, el conflicto y/o la lucha por el cambio sociales.

3. Una tercera alternativa sería la elaborada por E.O. Wright (1983), cuando ofrece el concepto de posiciones contradictorias dentro de las relaciones de clase como una solución al problema de las clases medias. Esta aproximación teórica permite concebir a la clase media como un grupo diferente con respecto a las otras clases sociales y con un estatus de clase social. En una obra posterior, el propio Wright (1994) propone una definición diferente de la clase media, recurriendo a una conceptualización extendida de la idea de propiedad o capital, para la cual dicha clase estaría dotada de recursos intelectuales y de organización, aunque no de la propiedad de los medios de producción.

4. Una cuarta solución puede ser encontrada en la obra de John Goldthorpe (Erikson y Goldthorpe, 1993), aunque el término clase media no sea el más recurrido, por no decir que está notablemente ausente. Sin embargo, su esquema de clases nos ofrece los elementos para extraer dos conclusiones importantes atingentes al problema que estamos tratando. Por un lado, la clase media conserva sus fronteras frente a la clase obrera, para lo cual se recurre a la distinción manual/no manual, siendo la clase obrera caracterizada por el segundo elemento de esta oposición. Sin embargo, por otro lado, si atendemos únicamente a las personas cuya subsistencia depende de vender su fuerza de trabajo, la clase media se divide en dos grandes conjuntos de clase, la clase de servicio, colindante con la élite propietaria, y el trabajo no manual de rutina. Sea como fuere, en este caso la clase media es el producto de un intento deliberado por clasificar a los individuos en base a principios o criterios relativos a las ocupaciones que aquéllos han alcanzado en su vida laboral presente.

\section{Solución empírica}

Existe un conjunto notable de autores que ha defendido que una solución al problema de la delimitación debe proceder del estudio de la realidad social y no puede derivarse de un análisis principalmente teórico de la misma, por exhaustivo que éste sea. Desde este punto de vista, se 
sostiene que la sociedad es demasiado compleja y cambiante, estando los grupos que la componen sometidos a un continuo proceso de formación, razón por la cual es fundamental recurrir a la evidencia empírica (Burris, 1986; Wacquant, en McNall, 1991; Crompton, 1992; Savage et al., 1995). Este es un punto importante para los propósitos del tema que estamos tratando en este artículo, dado que la sugerencia de acudir a los datos empíricos tiene como objetivo enfrentarse con la tarea de enjuiciar el grado de unidad real de una clase social cualquiera. Dentro de este conjunto de aportaciones, si bien existe consenso sobre estas tesis, cabe destacar que podemos encontrar también una significativa diversidad de ideas o énfasis entre los investigadores que han desarrollado esta línea argumental. En lo que sigue de esta sección, presentamos las que consideramos más relevantes.

1. Hablar de la formación de clase es una forma de reconocer la historicidad de la clase media y, por ello mismo, la imposibilidad de resolver el problema de su delimitación de una vez y para siempre (Wacquant, en McNall, 1991; Crompton, 1992). No obstante, conviene subrayar que desde la misma perspectiva pueden ser observadas la burguesía y la clase obrera, las cuales, según Wacquant (en McNall, 1991), son tratadas de forma a-problemática por casi todas las teorías de las clases medias.

2. Wacquant (en McNall, 1991) afirma que las regiones intermedias del espacio social, debido a su naturaleza, propenden a orientarse en direcciones opuestas y ello lleva a que la cuestión de las clases medias solamente pueda ser resuelta a un nivel ideológico y político, es decir, a través del estudio histórico de prácticas de clase reales ${ }^{14}$. A este respecto, parece hacerse eco de la postura bourdieuana (Bourdieu, 1979), que veía las posiciones intermedias como lugares de paso, es decir, sin verdaderos intereses o sin la consolidación de las posiciones dominantes y dominadas. En definitiva, las personas que ocupan posiciones en el medio del espacio social, según estas tesis, miran hacia arriba y hacia abajo tanto política como socialmente.

3. De acuerdo con Savage et al. (1995), para entender el proceso de formación de la clase media, debemos prestar atención a la relación que se produce en un lugar y momento concretos entre los tres recursos individuales que ellos consideran fundamentales desde el punto de vista de la clase social: la propiedad, la autoridad o posición en la jerarquía de la organización, y la cultura o educación. En este argumento recogen los criterios que Wright (1994) utiliza para la construcción de una de sus versiones de esquema de clases. Dejando de lado el interés que esto pueda tener en sí mismo, relativo al grado de vigencia de criterios marxistas para la definición de las clases sociales y de las clasificaciones en las que éstas se insertan, lo interesante aquí es observar como el problema de la delimitación se resuelve de dos modos distintos cuando en ambos casos se recurre a los mismos principios clasificadores. Ello se observa perfectamente si comparamos este punto con el tercero de la sección sobre las soluciones teóricas, la 5.1 . 
4. El recurso a lo empírico no sólo ha ido acompañado del desarrollo de propuestas teóricas menos centradas en los esquemas de clase, sino que ha servido para decidir sobre la pertinencia de algunos de los tipos derivados de estos últimos. Obviamente, solucionar el problema de la delimitación de las fronteras de clase recurriendo a lo empírico tiene esta finalidad. Un ejemplo de ello se encuentra en el trabajo de Szymanski (en Walker, 1979), quien concluye que debemos hablar de una única pequeña burguesía, con un componente viejo y otro nuevo. Por su parte, Val Burris (1986) rechaza que todos los empleados de cuello blanco, en general los trabajadores no manuales de oficina, puedan ser considerados miembros de una clase social bien cohesionada ${ }^{15}$. También se manifiestan en la misma línea Raynor (1970) y Garrard et al. (1978).

\section{Implicaciones del boundary problem}

Después de tratar la definición, las condiciones, las causas y las soluciones relativas al problema de la delimitación, es oportuno atender a las implicaciones del mismo, las cuales están estrechamente relacionadas con las cuestiones anteriormente mencionadas, pero sin reducirse a ellas, pues en este apartado se intenta insistir en la relevancia que ha tenido, tiene y tendrá la dificultad para trazar fronteras de clase nítidas y por todos aceptadas. Siguiendo la misma estructura de los apartados previos, listamos algunas de las implicaciones que son más significativas.

1. Independientemente de que el boundary problem sea insoluble, y quizás precisamente por serlo, es importante tratar con atención la definición de las clases sociales para evitar mal- y sobre-entendidos. No en vano, Crompton y Scott (en Crompton et al., 2000) afirman que algunos debates en sociología son pseudo-debates debido al uso de conceptos de clase diferentes. A este respecto, la clase media es un ejemplo palmario, pues los criterios según los cuales se define son multitud. En respuesta a ello, debemos, por un lado, hacer frente a los malentendidos, debidos a que podemos estar refiriéndonos a un grupo cuyas fronteras no son las que suponemos y ello nos puede llevar a conclusiones erróneas. En segundo lugar, por otro lado, debemos evitar los sobre-entendidos, y ello debe hacerse haciendo evidentes los criterios de acuerdo a los cuales a un conjunto de personas las denominamos clase media.

2. Si las clases sociales están en un proceso continuo de formación, su cristalización en un momento histórico y geográfico concreto no puede entenderse más que como un hecho cuya continuidad hacia el pasado y el futuro no podemos suponer. Ello obliga a adaptar los esquemas de clase a la realidad social y a definir fronteras de clase coyunturales. En otras palabras, más que ante un problema, estamos ante una condición que no tiene solución y se presenta como un reto para todo científico social que quiera aproximarse al estudio de la sociedad desde el punto de vista de las clases sociales. En otras palabras, podemos construir y emplear esquemas de clase, pero su vigencia será variable y siempre temporal. 
3. Pilbeam (1990) afirma que las definiciones de la clase media son variadas, ambiguas y vagas. Si observamos el punto 2 , inmediatamente anterior, es fácil entender por qué es así. En realidad, pudiera ser entendido como una cuestión de extensión e intensión conceptual ${ }^{16}$. Si queremos conceptos más extensos, que pueden ser utilizados en diferentes lugares y momentos históricos, manteniendo un significado similar, aunque lo sea lejanamente, podremos evitar la variedad, pero no la vaguedad $^{17}$. Si, por el contrario, recurrimos al empleo de conceptos más intensos, muy ajustados a su lugar y tiempo, la ambigüedad y la vaguedad desaparecerán o se verán disminuidas, pero se multiplicará la variedad de definiciones de clase media. En otros términos, dar satisfacción al punto 1 probablemente impedirá, o estará en tensión con, cumplir el 2, y viceversa.

4. Wahrman (1995) abunda en la idea de la vaguedad del referente social de la noción de clase media, y añade que a menudo esta imprecisión sirvió los propósitos de los que la utilizaron. Por tanto, la dificultad para trazar fronteras de clase puede favorecer el uso de conceptos borrosos de clase media con objetivos que dependerán de los intereses de aquéllos que los utilicen. El punto 1, es decir, una reflexión sobre la conceptualización de la clase media debería ayudar a corregir los excesos de vaguedad y eventualmente podría contribuir asimismo a descubrir las intenciones de los que promueven ciertas versiones. La idea de clase media ha sido históricamente un concepto políticamente cargado (Gayo, 2013) y eso no debería ser ocultado. Muy por el contrario, debiera ser tematizado e incorporado explícitamente en los análisis cuando sea oportuno.

5. Desde una perspectiva centrada claramente en lo político, E.O. Wright (1983) defiende que es importante saber cómo se conceptualizan las clases y qué categorías de posiciones sociales se sitúan dentro de las mismas, pues ello está estrechamente conectado con el desarrollo de una política socialista viable. Esto significa que el problema de la delimitación deviene un obstáculo relevante cuando dificulta conocer el referente social de cualquier tipo de proyecto político, es decir, desde el momento en que se constituye en un problema para la identificación de los referentes o grupos sociales en los cuales se apoyará aquél y a los cuales se querrá dar satisfacción mediante el mismo.

6. Por último, de acuerdo con Savage et al. (1995), el problema básico para escribir sobre las clases medias es encontrar una forma de ir más allá de definir a las mismas como un grupo heterogéneo que no puede ser incorporado ni a la clase trabajadora ni a la clase alta. Esta idea se refiere a la primera dificultad que mencionábamos en el apartado sobre la definición del problema de la delimitación (sección 2.1), y se deriva de la misma. De este modo, debería ser estudiado no sólo si la clase media es diferente a las otras clases, o si es un grupo homogéneo o heterogéneo, sino además, siendo fragmentada, con qué tipo de heterogeneidad nos encontramos. 


\section{Conclusiones: más allá del problema de la delimitación}

El problema de la delimitación puede ser interpretado en tres direcciones bien diferentes. Desde la primera, que podríamos llamar epistemológica, se pone en duda la utilidad de continuar haciendo uso de esquemas de clase teóricos como medio de hacer comprensible la realidad social. Desde la segunda interpretación, que denominaremos empírica, lo que se cuestiona no es el empleo de las clasificaciones de clase, sino su deducción desde principios teóricos y su empleo más allá de donde eventualmente emergieron de forma significativa. Esto incluye una advertencia: los esquemas de clase deben referirse a las sociedades estudiadas. Una tercera vía interpretativa, a la que aquí llamaremos teórica, es la que apuesta por seguir trabajando para construir y en su caso perfeccionar los esquemas de clase, dados los importantes beneficios científicos que esta forma de análisis social ha permitido obtener. Las dos últimas interpretaciones conservan el interés en el análisis de clase, y, aunque sus estrategias intelectuales sean diferentes, ambas enfrentan con fuerza el problema del delineamiento de fronteras de clase.

Sin embargo, sería limitado pensar que estamos ante una dificultad que afecta principal o únicamente a los investigadores que han adoptado o quieren adoptar un enfoque de clase para el estudio de los fenómenos sociales, y en particular a aquéllos interesados principalmente en la clase media. Muy por el contrario, aunque ello parezca extraño, el boundary problem no es necesariamente un problema, y hasta podría decirse que no se refiere únicamente al trazado de fronteras de clase. En realidad, es un síntoma de la deriva de una sociología fuertemente teórica, cuantitativa, omniabarcante, con aspiraciones comparativas y con ello con una clara tendencia a la ubicuidad. El problema mencionado sirvió para denunciar una forma de hacer sociología, que parecía cada vez más distante de la sociedad que decía representar, lo cual implicaba un creciente desconocimiento de las distinciones que en la dinámica de la misma los individuos generan. 


\section{Notas}

${ }^{1}$ Aquí me estoy refiriendo a la relación entre política y clase, o, en otros términos, a la clase como una variable fundamental para entender el comportamiento individual y los acontecimientos políticos. Para una revisión extensa de esta temática en la tradición sociológica, véase Gayo (2013).

${ }^{2}$ Para una breve revisión histórica del uso de la idea de clase media en Latinoamérica, puede verse el capítulo 1 del libro de Franco et al. (2010), escrito por este mismo autor junto a Martín Hopenhayn.

${ }^{3}$ Ese futuro es quizás para nosotros el pasado, una promesa en desarrollo desde los años 50 del siglo XX (Gayo, 2013). También es el presente, y se concreta en la problemática sobre la relación entre clase media y democracia, que efectivamente tiene décadas de vigencia, como apuntábamos antes, pero sigue siendo un tema de actualidad (Chauvel, 2006). Asimismo, para una reflexión más ligada a la globalización, la cual también atiende al vínculo entre clase media y estabilidad institucional, véase López y Weinstein (2012).

${ }^{4}$ En relación con la identificación con la clase media, para Chile pueden verse las aportaciones de Espinoza y Barozet (2009) y Barozet y Fierro (2011), y para Francia la de Bosc (2008). Un estudio que muestra la diversidad de este fenómeno, muy generalizado en los Estados Unidos y restringido en Inglaterra, es el de Devine (2011).

${ }^{5}$ Desde mi punto de vista, esto es precisamente lo que no sucede cuando se recurre a los ingresos como elemento definitorio de la incorporación a la clase media, como podemos constatar en la contribución de León et al. (Franco et al., 2010). Este es el abordaje típico de los economistas, los cuales parecen sentirse más cómodos cuando la evolución de la sociedad se entiende como el estudio de las transformaciones en los ingresos de las personas, sea esto entendido desde los individuos o desde los hogares.

${ }^{6}$ Un buen ejemplo de estas aproximaciones, con un énfasis claro en elementos culturales, puede encontrarse en el trabajo de Méndez (2008, 2010).

${ }^{7}$ En relación con el consumo cultural en Chile, en Gayo et al. (2012) puede encontrarse un ejercicio de construcción de clases sociales a partir del comportamiento manifiesto.

${ }^{8}$ Para una revisión de este debate, tratado desde la temática de la heterogeneidad o fragmentación de la clase media, véase Méndez y Gayo (2007).

${ }^{9}$ Yendo más allá, tras realizar un ejercicio estadístico a través del cual intentan identificar empíricamente a la clase media chilena, Espinoza y Barozet (2009). concluyen que es muy difícil encontrar a un grupo de personas con características compartidas a las que podamos atribuir la idea de clase media sin dificultades.

${ }^{10}$ Howe afirma que The boundary question has its significance in the ability of class analysis to identify the potential for unity within highly segmented and diversified classes (1992: p.95).

${ }^{11}$ the new middle class has stubbornly resisted all attempts to define its upper and lower limits (Dahrendorf, 1969: p.52). 
12 Este autor afirma que Fréquemment usités despuis le début du XIXe siècle, les vocables “classe moyenne" ou <"classes moyennes" ont connu des évolutions sémantiques considérables qui tiennent à lambiguïté de lexpression (Bosc, 2008: p.6).

${ }^{13}$ En el libro de Bouffartigue et al. (2011) hay varios capítulos dedicados a lo que en Francia se entiende como cadres, los cuales son un estrato de posiciones directivas, y se estudia como esta es una categoría cuya constitución y supervivencia está asociada a dinámicas de lucha y conflicto.

${ }^{14}$ Como un ejemplo de este punto, me parece ilustrativa la siguiente cita de Loic J. D. Wacquant refiriéndose a este problema: it cannot be adequately addressed at an abstract theoretical level and should thus be tackled, if at all, through historical analysis (McNall et al., 1991, capítulo 3: pp.39-40).

${ }^{15}$ What is not consistent with the empirical evidence is any theory that treats all whitecollar employees as members of a single cohesive class (Burris, 1986: p.34).

${ }^{16}$ Los conceptos de extensión e intensión son definidos por Sartori (1970) del siguiente modo: the extension (denotation) and intension (connotation) of a term. A standard definition is as follows: The extension of a word is the class of things to which the word applies; the intension of a word is the collection of properties which determine the things to which the word applies. Likewise, the denotation of a word is the totality of objects indicated by that word; and the connotation is the totality of characteristics anything must possess to be in the denotation of that word (p. 1041).

${ }^{17}$ Sartori (ídem) afirma que the net result of conceptual straining is that our gains in extensional coverage tend to be matched by losses in connotative precision. It appears that we can cover more in travelling terms only by saying less, and by saying less in a far less precise manner (p.1035). 


\section{Bibliografía}

Abercrombie, Nicholas. y Urry, John (1983), Capital, Labour and the Middle Classes, George Allen \& Unwin, London.

Bauman, Zygmunt (2001), The Individualized Society, Polity, Cambridge.

Bauman, Zygmunt (2007), Vida de Consumo, Fondo de Cultura Económica, Buenos Aires.

Beck, Ulrich y Beck-Gernsheim, Elisabeth (2002), Individualization, Sage, London.

Barozet, Emmanuelle y Fierro, Jaime (2011), Clase media en Chile, 19902011: algunas implicancias sociales y políticas, Serie de Estudios, Fundación Konrad Adenauer, ${ }^{\circ}$ 4, Santiago.

Bonham, John (1954), The Middle Class Vote, Faber and Faber, London.

Bosc, Serge (2008), Sociologie des clases moyennes, La Découverte, Paris.

Bourdieu, Pierre (1979), La distinction, les Éditions de Minuit, Paris.

Bouffartigue, Paul, Gadea, Charles y Pochic, Sophie (directores) (2011), Cadres, clases moyennes: vers léclatement, Armand Colin, Paris.

Braverman, Harry (1974), Labour and Monopoly Capital: the Degradation of Work in the Twentieth Century, Monthly Review Press, New York.

Brint, Steven (1985), “The political attitudes of professionals”, Annual Review of Sociology, 11: pp.389-414.

Brooks, Clem y Manza, Jeff (1997), "Partisan Alignments of the 'Old' and 'New' Middle Classes in Post-Industrial America”, en Clark, Terry Nichols y Rempel, Michael, Citizen politics in post-industrial societies, Boulder, Westview: pp.143-157.

Burris, Val (1986), "The discovery of the new middle class", Theory and Society, 15: pp.317-349.

ídem (1987), “Class Structure and Political Ideology”, The Insurgent Sociologist, Vol.14, n.2: pp.5-46.

Butler, Tim y Savage, Mike (1995), Social Change and the Middle Classes, University College London Press, London.

Callinicos, Alex y Harman, Chris (1987), The Changing Working Clas, Bookmarks, London. 
Carabaña, Julio y Francisco, Andrés de (eds.) (1994), Teorías Contemporáneas de las clases sociales, Ed. Pablo Iglesias, Madrid.

Chauvel, Louis (2006), Les classes moyennes à la dérive, Éditions du Seuil et La République des Idées.

Crompton, Rosemary (1992), "Patterns of Social Consciousness amongst the Middle Classes”, en Burrows, Roger \& Marsh, Catherine (eds.), Consumption and Class: Divisions and Change, St. Martin's Press (Macmillan, London): pp.140-165, New York.

Crompton, Rosemary; Devine, Fiona; Savage, Mike y Scott, John (eds.) (2000), Renewing Class Analysis, Blackwell Publishers/ The Sociological Review, Oxford.

Dahrendorf, Ralf (1969), Class and Class Conflict in Industrial Society, Routledge \& Kegan Paul, London.

Devine, Fiona (1997), Social Class in America and Britain, Edinburgh University Press, Edinburgh.

Devine, Fiona (2011), Upper-middle ou lower-middle? Classes moyennes et identité de classe aux USA et en Angleterre, en Bouffartigue, Paul, Gadea, Charles y Pochic, Sophie (directores), Cadres, clases moyennes: vers léclatement, Armand Colin: pp.275-286, Paris.

Eder, Klaus (1995), “Does Social Class Matter in the Study of Social Movements? A Theory of Middle-Class Radicalism”, in Maheu, L. (ed.): Social movements and social classes, Sage: pp.21-54, London.

Erikson, Robert y Goldthorpe, John H. (1993), The Constant Flux. A Study of Class Mobility in Industrial Societies, Clarendon Press, Oxford.

Espinoza, Vicente y Barozet, Emmanuelle (2009), “¿De qué hablamos cuando decimos clase media? Perspectivas sobre el caso chileno”, en Joignant, Alfredo y Güell, Pedro (coords.), El arte de clasificar a los chilenos. Enfoques sobre los modelos de estratificación en Chile, Universidad Diego Portales, capítulo 5: pp.103-130, Santiago.

Franco, Rolando; León, Arturo y Atria, Raúl (coords.) (2007), Estratificación y movilidad social en América Latina. Transformaciones estructurales de un cuarto de siglo, LOM ediciones, Santiago.

Franco, Rolando; Hopenhayn, Martín y León, Arturo (coords.), Las clases medias en América Latina, Siglo XXI, CEPAL, México D.F.

Garrard, John; Jarry, David; Goldsmith, Michael \& Oldfield, Adrian (eds.) (1978), The Middle Class in Politics, Saxon House, England. 
Gayo, Modesto; Teitelboim, Berta; y Méndez, María Luisa (2013), “Exclusividad y fragmentación: los perfiles culturales de la clase media alta en Chile”, revista Universum, Universidad de Talca, n 28, Vol. 1: pp.97-128.

Gayo, Modesto; Teitelboim, Berta; y Méndez, María Luisa (2012), Desigualdad cultural y estructura ocupacional. La pos-industrialización en chile. artículo no publicado.

Gayo, Modesto (2013), "Revisiting middle class politics: a multidimensional approach”. Evidence from Spain, The Sociological Review, Vol. 61: pp.814837.

Gunn, Simon (2004), “Cultural Capital and the English Middle Classes in Historical Perspective”, borrador presentado en el Cultural Capital and Social Exclusion Symposium, Oxford (Reino Unido), 8-10 enero.

Hanlon, Gerard (1998), "Professionalism as enterprise: service class politics and the redefinition of professionalism”, Sociology, vol.32, n.1, February: pp.43-63.

Howe, Carolyn (1992), Political Ideology and Class Formation: A Study of the Middle Class, Praeger, Westport, Connecticut,.

Inglehart, Ronald (1991), El cambio cultural en las sociedades industriales avanzadas, Siglo XXI, CIS, Madrid.

López, A. Ricardo y Weinstein, Barbara (2012): Introduction: We Shall Be All: Toward a Transnational History of the Middle Class, en libro editado por los mismos autores, The Making of the Middle Class. Toward a Transnational History, Duke University Press, Durham.

McNall, Scott G.; Levine, Rhonda F. \& Fantasia, Rick (1991), Bringing Class Back In. Contemporary and Historical Perspectives, Westview Press, Boulder.

Méndez, María Luisa y Gayo, Modesto (2007), “El perfil de un debate: movilidad y meritocracia. Contribución al estudio de las sociedades latinoamericanas”, en Franco, R., León, A. y Atria, R. (coords.), Estratificación y movilidad social en América Latina. Transformaciones estructurales de un cuarto de siglo, LOM ediciones, capítulo III: pp.121-157, Santiago.

Méndez, María Luisa (2008), “Middle class identities in a neoliberal age: tensions between contested authenticities”, The Sociological Review 56(2): pp.220-237.

Méndez, María Luisa (2010), “Las clases medias en Chile: transformaciones, sentido de pertenencia y tensiones entre distintos proyectos de movilidad”, en Rolando Franco, Martín Hopenhayn y Arturo León (coords.), 
Las clases medias en América Latina, Siglo XXI, CEPAL, capítulo 5: pp.230-288, México D.F..

Mills, C. Wright (1959), White Collar. The American Middle Classes, a Galaxy Book, Oxford University Press, New York.

Pilbeam, Pamela M. (1990), The middle classes in Europe 1789-1914: France, Germany, Italy and Russia, Macmillan, London.

Poulantzas, Nicos (1974), Les classes sociales dans le capitalisme aujourd'hui, Editions du Seuil, Paris.

Raynor, John (1970), The Middle Class, Longman, London.

Sartori, Giovanni (1970), “Concept Misformation in Comparative Politics”, The American Political Science Review, vol. 64, n. 4, diciembre: pp.10331053.

Savage, Mike; Dickens, Peter \& Fielding, Tony (1988), “Some social and political implications of the contemporary fragmentation of the "service class' in Britain”, International Journal of Urban \& Regional Research, 12, 3, 1988: pp.455-476.

Savage, Mike; Barlow, James; Dickens, Peter \& Fielding, Tony (1995), Property, Bureaucracy and Culture. Middle-Class Formation in Contemporary Britain, Routledge, London.

Vidich, Arthur J. (ed.) (1995), The New Middle Classes. Life-Styles, Status Claims and Political Orientations, Macmillan, London.

Wahrman, Dror (1995), Imagining the Middle Class. The Political Representation of Class in Britain, c.1780-1840, Cambridge University Press, Cambridge.

Walker, Pat (1979), Between Labour and Capital, The Harvester Press, Sussex.

Wright, Erik Olin (1983), Clase, crisis y estado, Siglo XXI, Madrid.

Wright, Erik Olin (1994), Clases, Siglo XXI, Madrid.

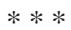

Recibido: 04.11.2011

Aceptado: 10.04.2013 